\title{
Smooth Muscle Hyperplasia in an Asthmatic Patient: Do We Know It All?
}

\author{
Begum Ergan-Arsava ${ }^{a}$ Gul Karakaya ${ }^{b}$ Pinar Firat ${ }^{c}$ A. Fuat Kalyoncu ${ }^{b}$

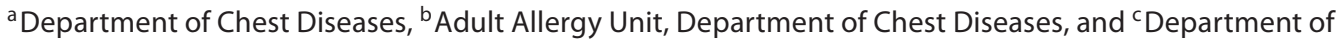 \\ Pathology, Hacettepe University, Faculty of Medicine, Ankara, Turkey
}

\section{Established Facts}

- Smooth muscle hyperplasia is an important component of structural airway pathology in asthma. However, the diagnosis of smooth muscle hyperplasia/hypertrophy in asthmatic patients is very challenging. Most of the current knowledge regarding this issue comes from postmortem studies.

\section{Novel Insights}

- The present contribution describes a patient with analgesic-induced asthma, who was diagnosed with an endobronchial mass caused by extensive smooth muscle proliferation which morphologically resembled florid hyperplasia. We consider the case impressive because it represents an extreme example of benign smooth muscle hyperplasia forming an endobronchial mass in a living asthma patient.

\section{Key Words}

Adult asthma - Airway smooth muscle $\cdot$ Endobronchial polypoid mass $\cdot$ Smooth muscle hyperplasia

\begin{abstract}
Bronchial smooth muscle hyperplasia is a well-known structural change in asthma. The degree of hyperplasia is related to asthma severity. We report a case of extreme smooth muscle hyperplasia in an asthmatic patient. A 54-year-old female with a diagnosis of analgesic-induced asthma was admitted
\end{abstract}

to our center for nasal polyposis surgery. During her preoperative evaluation, atelectasis of the right middle lobe was detected on chest X-ray. Bronchoscopy revealed the presence of a vegetating polypoid mass obliterating the entrance of the right middle lobe. Histopathological examination of the surgically excised polypoid mass showed benign smooth muscle proliferation with diffuse eosinophilic infiltration. This is a rare case representing an extreme example of benign smooth muscle hyperplasia forming an endobronchial mass in an asthmatic patient.

Copyright $\odot 2010$ S. Karger AG, Basel

\section{KARGER \\ Fax +41613061234 E-Mail karger@karger.ch} www.karger.com
Begum Ergan-Arsava, MD

Department of Chest Diseases

Hacettepe University, Faculty of Medicine

TR-06100 Sihhiye, Ankara (Turkey)

Tel. +90 31230515 31, Fax +90 31231008 09, E-Mail erganb@ hacettepe.edu.tr 


\section{Introduction}

The pathologic role of airway smooth muscle (ASM) in asthma has been known for years. An increased ASM mass plays an important role in the remodeling process $[1,2]$. The amount of ASM proliferation in asthmatic subjects is difficult to evaluate; however, previous studies showed a marked increase in ASM mass especially in fatal asthma cases [1]. An autopsy study performed by Ebina et al. [3] demonstrated two types of ASM proliferation: type 1 in which the ASM mass increased only in the central bronchi, and type 2 in which the ASM mass increased throughout the bronchial tree. We report an extreme case of ASM proliferation leading to mass formation and bronchial obstruction in a patient with analgesic-induced asthma.

\section{Case Report}

A 54-year-old nonsmoker female was hospitalized for nasal polyposis surgery. She had a diagnosis of bronchial asthma and perennial rhinitis since the age of 31 . As she developed acute urticaria and asthma symptoms after consumption of aspirin and metamizol, she was diagnosed with analgesic-induced asthma (analgesic-exacerbated respiratory disease) in our center. Her skin prick test was negative for common aeroallergens. Her asthma had been corticosteroid-dependent since her diagnosis; combination therapy of low-dose inhaled steroid and $\beta$-mimetic (budesonide $160 \mu \mathrm{g}$ and formeterol fumarate $4.5 \mu \mathrm{g}$ once daily) was initiated a year ago as her symptoms became mild persistent. During her follow-up in our institution, she had two nasal polypectomy surgeries for symptomatic relief of nasal passage obstruction due to nasal polyps.

On the preoperative evaluation during her current admission, her physical examination revealed no abnormal findings. A paranasal computerized tomography demonstrated sinonasal polyposis with inflammatory opacification in the frontal, ethmoid, maxillary and sphenoidal sinuses. Complete blood count revealed mild eosinophilia. Erythrocyte sedimentation rate, serum electrolytes, liver and renal function tests were within normal limits. Pulmonary function tests revealed a forced expiratory volume in $1 \mathrm{~s}\left(\mathrm{FEV}_{1}\right)$ of 1.65 liters (76\% of predicted), forced vital capacity (FVC) of 2.57 liters (100\% of predicted) and an $\mathrm{FEV}_{1} / \mathrm{FVC}$ ratio of 64\%. Previous pulmonary function test results were: $\mathrm{FEV}_{1}, 1.11$ liters (60\% of predicted), FVC, 1.59 liter (72\% of predicted) and $\mathrm{FEV}_{1} / \mathrm{FVC}, 69 \%$. A reticulonodular opacity in the right lower lung zone was detected on the posteroanterior chest X-ray (CXR), which was confirmed to be secondary to total atelectasis of the right middle lobe by a right lateral CXR (fig. 1a). Reevaluation of her previous CXRs confirmed that the right middle lobe total atelectasis was a new finding. A computerized tomography scan of the thorax showed right middle lobe bronchus obliteration with total atelectasis of the distal parenchyma (fig. 1b). Fiberoptic bronchoscopy demonstrated total obliteration of the entrance of the right middle lobe by a mucous plug and an underlying vegetat- ing polypoid mass. Pathologic examination of the forceps biopsy specimen from the lesion showed diffuse eosinophilic infiltration with fibrosis under the bronchial mucosa and vascular proliferation. A second biopsy, obtained by rigid bronchoscopy, confirmed the findings of the previous biopsy specimen but also revealed squamous metaplasia and smooth muscle proliferation. As a definitive diagnosis could not be made, surgery was planned and right middle lobectomy with lymph node dissection was performed. Macroscopic examination of the pathological specimen showed a polypoid mass $2.5 \mathrm{~cm}$ in diameter totally obstructing the right middle lobe bronchus. Histopathological examination of the lesion revealed florid smooth muscle proliferation admixed with dense eosinophilic infiltration (fig. $2 a-c$ ). The margins of the lesion were ill defined; it did not show pushing margins and there was no surrounding capsule. There was no evidence of cellular atypia or pleomorphism. There were submucosal glands embedded within the ASM cells (fig. 2b). The tissue architecture of the submucosal area was fairly intact, with proliferating smooth muscle growing within, but not replacing or destructing the normal structures, which suggested a morphologic diagnosis of florid hyperplasia. Immunohistochemical studies showed positive staining with smooth muscle actin and desmin (fig. 2d); S-100, CD34, pan-cytokeratin stains were negative. Further molecular studies searching for the presence of clonality to rule out a neoplastic origin could not be performed. The increase in smooth muscle mass was only present around the lobar bronchus as a continuation of the lesion. Resection margins were intact and all excised lymph nodes were found to be reactive. The whole bronchial tree was heavily infiltrated by eosinophils; eosinophils were present in the mucosa, submucosa, within the muscle bundles and in the lumen of the bronchi.

The final diagnosis was abnormal smooth muscle proliferation mostly in favor of hyperplasia protruding into the right middle lobe bronchus. After her recovery from thoracotomy, she had nasal polypectomy without any complications. A follow-up fiberoptic bronchoscopy, which was performed 2 years after surgery, revealed normal findings except for scar formation in the orifice of the right middle lobe. Currently, 4 years after surgery, she is doing well without any complaints.

\section{Discussion}

Asthma is a chronic inflammatory disease characterized by hyperresponsive airways. In addition to the inflammatory response, there are structural changes in the asthmatic airways, such as goblet cell hyperplasia, angiogenesis, subepithelial fibrosis and an increase in ASM mass. These changes are known as airway remodeling [2]. Although traditionally ASM hyperplasia and hypertrophy are thought to be a consequence of ongoing inflammation, it is now known that ASM has an important regulatory role in airway inflammation [4]. ASM is responsible for the release of extracellular matrix proteins, which lead to basement membrane thickening and increased fibrosis [4]. In addition, ASM cells release growth 

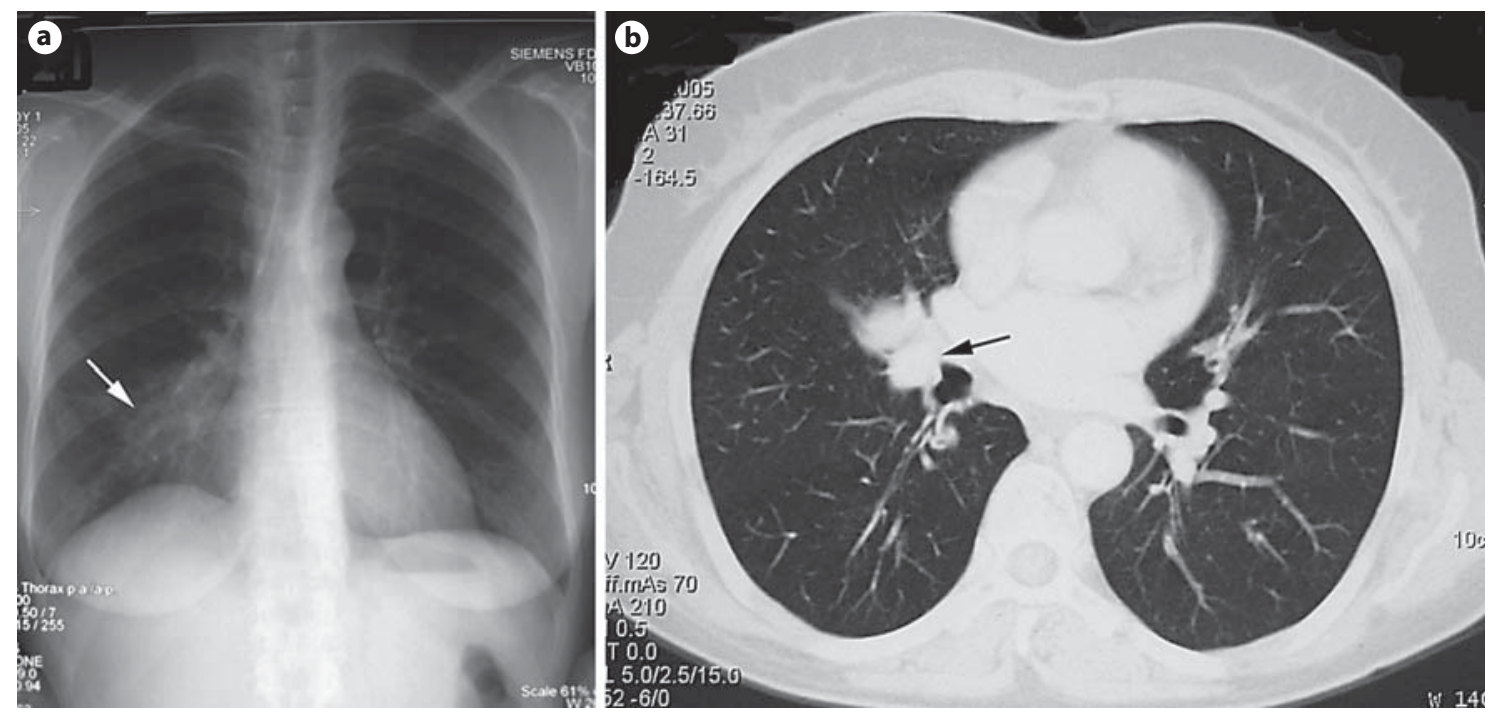

Fig. 1. a Posteroanterior CXR showing right middle lobe atelectasis (arrow). b Chest computerized tomography scan showing right middle bronchus obstruction (arrow) and atelectasis of distal lung parenchyma.

factors, proangiogenetic factors and other cytokines, and also interact with inflammatory cells [5]. Because of these properties, ASM is not only the target of many conventional asthma treatments, such as corticosteroids and $\beta_{2}$-agonists, but also of new interventions like thermoplasty $[4,6]$.

The ASM mass increase may be secondary to hypertrophy and/or hyperplasia [3,7-10]. This process is usually related to asthma severity; ASM mass is estimated to increase by a factor of $50-200 \%$ in nonfatal asthma whereas the increase is in the range of $200-400 \%$ in fatal asthma [11]. In our case, asthma had been corticosteroiddependent for a long time. Therefore, the length and severity of asthma in this case might have contributed to ASM hyperplasia, consistent with the close relationship between an increased ASM mass and asthma severity [1, $7,8]$.

In this case, the morphological features of the histopathological specimen were suggestive of ASM hyperplasia. The tissue architecture of the submucosal area was intact; proliferating muscle cells did not displace or compress the adjacent structures and there was no evidence of cellular atypia. Despite all these features suggestive of a nonneoplastic origin, molecular analyses which would have given further information on clonality could not be performed, and therefore a neoplastic process - such as leiomyoma - could not be definitely ruled out [12].
An important feature of the histologic examination of the surgical specimen was the presence of diffuse and dense eosinophilic infiltration in the lesion, highlighting the presence of an extensive inflammatory component. Previous literature has shown the presence of a close interaction between ASM and inflammatory cells, including eosinophils $[5,13]$. Inflammatory cells were found to be associated with induction of ASM proliferation. In addition, the patient also had recurrent nasal polyps, which are known to be closely linked to excessive chronic mucosal inflammation. Polyp tissue typically contains a predominance of eosinophils in analgesic-intolerant patients [14]. We therefore believe that the chronic eosinophilic infiltration might have contributed to the excessive ASM hyperplasia observed in this patient.

In this case, the diagnosis was established after the incidental detection of total atelectasis of the right middle lobe in the CXR during a routine preoperative evaluation. Although an endobronchial lesion in an asthmatic patient may cause worsening of symptoms, surprisingly in this case, asthma was well controlled as confirmed by pulmonary function tests. Local signs of partial obstruction on auscultation, such as localized rhonchi, may facilitate the diagnosis of endobronchial lesions. Even though no signs suggestive of endobronchial obstruction were present in our patient, the detection of such localized signs could easily have been attributed to her primary disease, asthma. Therefore, we think that local aus- 

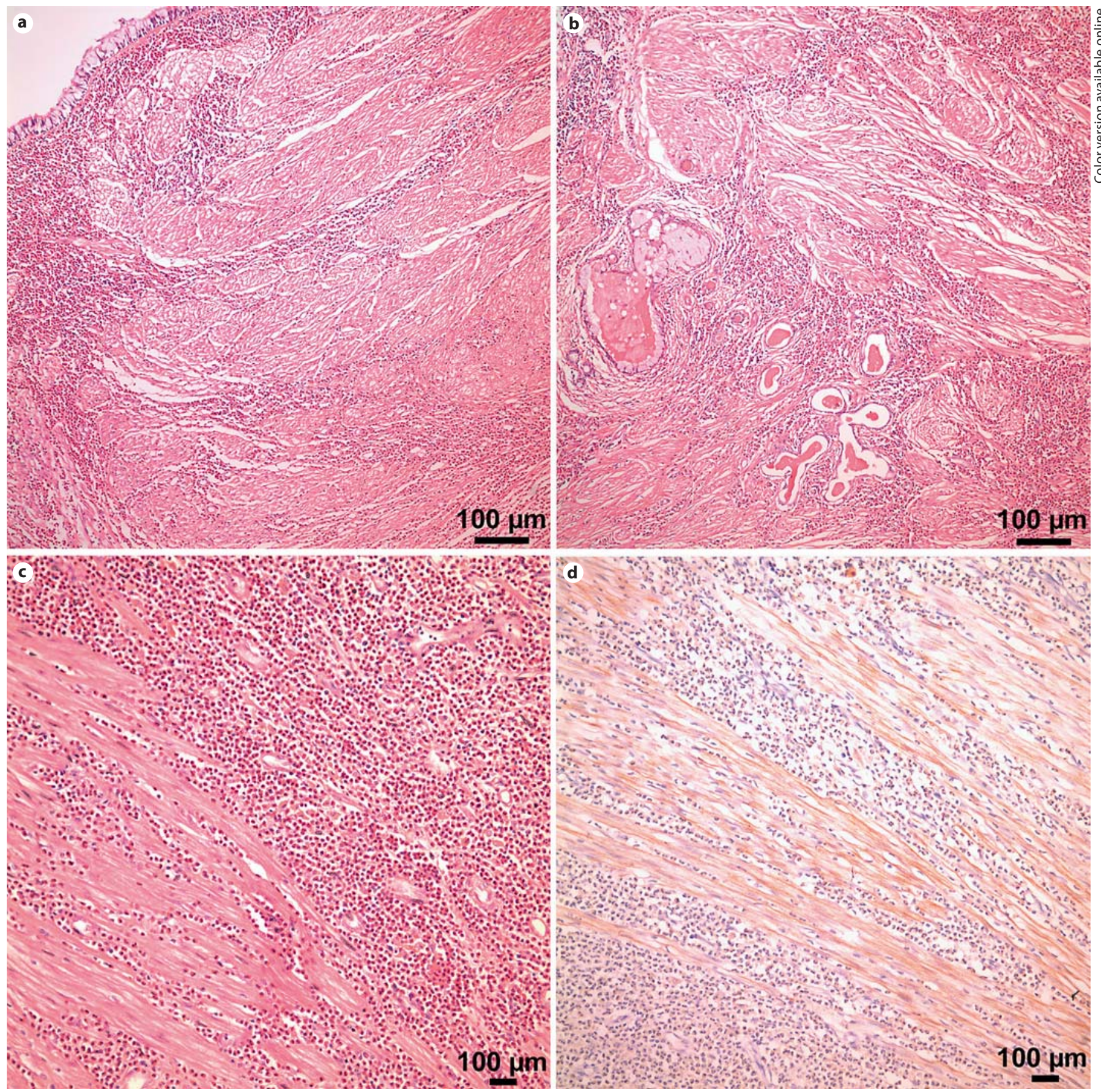

Fig. 2. a, b Diffuse smooth muscle proliferation involving submucosal area (HE). $\times 100$. c Eosinophilic infiltration intermingled with smooth muscle cells (HE). $\times 200$. d Desmin-stained smooth muscle cells. $\times 200$.

cultation findings or findings refractory to steroid treatment in an asthmatic patient should be evaluated with care, as these may point to the presence of a new pathology. Additional diagnostic investigations such as computerized tomography and/or fiberoptic bronchoscopy should be performed promptly in case of clinical suspicion.

In conclusion, ASM hyperplasia is an important component of airway pathology in asthma. However, the diagnosis of ASM hyperplasia/hypertrophy in asthmatic 
patients can be very challenging. First of all, bronchial biopsy is rarely indicated in asthmatic patients. Second, fiberoptic bronchoscopy is considered as a high-risk procedure in patients with severe asthma, who are expected to have a significant increase in ASM mass. Third, technical difficulties in obtaining a full-thickness mucosal bi- opsy and disparities in tissue processing might lead to variations in ASM mass quantification [15]. Because of all these reasons, knowledge about the extent of ASM hyperplasia in living asthma patients is limited and our case, which is interpreted as ASM hyperplasia forming an endobronchial mass in an asthmatic patient, is thus unique.

\section{References}

1 Bentley JK, Hershenson MB: Airway smooth muscle growth in asthma: proliferation, hypertrophy, and migration. Proc Am Thorac Soc 2008;5:89-96.

2 Global Initiative for Asthma: global strategy for asthma management and prevention. Available at: http://www.gianasthma.org.

-3 Ebina M, Takahashi T, Chiba T, Motomiya M: Cellular hypertrophy and hyperplasia of airway smooth muscles underlying bronchial asthma. A 3-D morphometric study. Am Rev Respir Dis 1993;148:720-726.

4 Oliver BG, Black JL: Airway smooth muscle and asthma. Allergol Int 2006;55:215-223.

5 Hirst SJ: Regulation of airway smooth muscle cell immunomodulatory function: role in asthma. Respir Physiol Neurobiol 2003;137: 309-326.

-6 Castro M, Rubin AS, Laviolette M, Fiterman J, De Andrade Lima M, Shah PL, Fiss E, Olivenstein R, Thomson NC, Niven RM, Pavord ID, Simoff M, Duhamel DR, McEvoy C, Barbers R, Ten Hacken NH, Wechsler ME, Holmes M, Phillips MJ, Erzurum S, Lunn W, Is- rael E, Jarjour N, Kraft M, Berry SM, Quiring J, Shargill NS, Cox G: Effectiveness and safety of bronchial thermoplasty in the treatment of severe asthma: a multicenter, randomized, double-blind, sham-controlled clinical trial. Am J Respir Crit Care Med 2010;181:116-124.

7 Woodruff PG, Dolganov GM, Ferrando RE, Donnelly S, Hays SR, Solberg OD, Carter R, Wong HH, Cadbury PS, Fahy JV: Hyperplasia of smooth muscle in mild to moderate asthma without changes in cell size or gene expression. Am J Respir Crit Care Med 2004; 169:1001-1006.

$\checkmark 8$ Benayoun L, Druilhe A, Dombret MC, Aubier M, Pretolani M: Airway structural alterations selectively associated with severe asthma. Am J Respir Crit Care Med 2003; 167:1360-1368.

9 Johnson PR, Roth M, Tamm M, Hughes M, Ge Q, King G, Burgess JK, Black JL: Airway smooth muscle cell proliferation is increased in asthma. Am J Respir Crit Care Med 2001; 164:474-477.
10 Hossain S, Heard BE: Hyperplasia of bronchial muscle in chronic bronchitis. J Pathol 1970;101:171-184.

11 Bai TR, Knight DA: Structural changes in the airways in asthma: observations and consequences. Clin Sci 2005;108:463-477.

12 Patton KT, Cheng L, Papavero V, Blum MG, Yeldandi AV, Adley BP, Luan C, Diaz LK, Hui $\mathrm{P}$, Yang XJ: Benign metastasizing leiomyoma: clonality, telomere length and clinicopathologic analysis. Mod Pathol 2006;19: 130-140.

13 Chung KF: Airway smooth muscle cells: contributing to and regulating airway mucosal inflammation? Eur Respir J 2000;15:961968.

14 Drake-Lee AB, McLaughlan P: Clinical symptoms, free histamine and $\operatorname{IgE}$ in patients with nasal polyposis. Int Arch Allergy Appl Immunol 1982;69:268-271.

15 James A, Carroll N: Airway smooth muscle in health and disease; methods of measurement and relation to function. Eur Respir J 2000;15:782-789. 